\title{
Male reproductive ageing: a tale of the whole ejaculate
}

\author{
Claudia Fricke and Mareike Koppik ${ }^{+}$ \\ Institute for Evolution and Biodiversity, Münster University, Münster, Germany \\ Correspondence should be addressed to C Fricke; Email: Claudia.Fricke@uni-muenster.de \\ ${ }^{\dagger}(M$ Koppik is now at Department of Ecology and Genetics, Uppsala University, Uppsala, Sweden)
}

\begin{abstract}
Ageing is nearly ubiquitous and encompasses all biological functions. We here focus on age-dependent changes in male reproductive capacity across a broad range of animal taxa. While there has been a long-standing focus on mating ability and overall reproductive success, we here highlight the underlying mechanisms that explain loss in fertilisation capacity in ageing males. Fertilisation is mediated by not only the presence of sperm, but also the cocktail of seminal fluid proteins that ensure sperm survival, capacitation and interaction with female physiology. Sperm ageing has received much attention in studies of male reproductive senescence; however, post-mating processes include a number of interlocked steps that together cumulate in successful fertilisation. As such we consider male ability to elicit female post mating responses such as uterine conformational changes, sperm storage and ovulation and the components within the ejaculate that mediate these post-mating processes. For the latter seminal fluid proteins are key and hence we reflect on age-dependent changes in quality of the entire ejaculate and its consequences for male reproductive capacity. While first studies accrue and highlight that changes in the non-sperm fraction can explain substantial variation in senescent male reproductive success and male ability to induce post-mating responses necessary for fertilisation many open questions still remain that warrant further investigations. One being what the potential age-dependent changes in composition are or whether there is a general decline and how this interacts with sperm to affect fertilisation success. Further, the impact females might have to ameliorate these changes will be an area of interest.

Reproduction (2019) 158 R219-R229
\end{abstract}

Ageing is a near ubiquitous phenomenon and observed as a decline in survival and reproductive performance with age (Nussey et al. 2013, Jones et al. 2014, Lemaitre et al. 2015, Maklakov \& Immler 2016, Lemaitre \& Gaillard 2017). The decline in survival (=actuarial ageing) is observed in a large number of organisms, both in the wild and in captivity (Nussey et al. 2013, Lemaitre et al. 2015). Its genetic basis as well as factors influencing the rate of ageing have been studied widely, with a whole body of theory explaining this phenomenon (for e.g. by Maklakov \& Immler 2016, Gaillard \& Lemaitre 2017, Lemaitre \& Gaillard 2017). In this review we focus on age-dependent changes in male reproduction and the factors that affect the onset and rate of these changes. We have reviewed recent examples (from the past 10 years) across several animal taxa ranging from invertebrates to vertebrate species studied both in the wild and the laboratory. We focus on studies reporting senescent changes in the ejaculate, the tissues producing the ejaculate and the consequences for male reproductive success.

Reproductive senescence is characterised by a decline in sexual trait expression coupled with diminishing reproductive returns. Similar to actuarial ageing, reproductive ageing has also been observed in a large number of taxa and occurs in the wild, but here it is mainly studied in mammals and birds (reviewed in Lemaitre \& Gaillard 2017, see Rodriguez-Munoz et al. 2019 for an insect example). It can be measured as a decline in mating activity and fertilisation success with increasing age and is often due to somatic changes, like decreased hormonal levels leading to increased dysfunction or less agile courtship resulting in lost mating opportunities. To give some brief examples, in fowl Gallus gallus, older males are less aggressive, resulting in fewer matings and reduced reproductive success as aggression is one important determinant of male mating success (McDonald et al. 2017). Apart from behaviour, a reduced investment in sexual ornaments, as observed in the decreased comb size in ageing fowl (Cornwallis et al. 2014) or duller foot colour in the blue-footed booby Sula nebouxii (Velando et al. 2011), can result in active discrimination by females. Senescent blue-footed booby males potentially signal their lower reproductive values, as those who carry more damaged DNA sites in their sperm also display a duller foot colour (Velando et al. 2011). However, a decline in mating performance is not inevitable or might be context dependent. Ageing male houbara bustards (Chlamydotis undulata) show no decline in sexual displays (Preston et al. 2011) and older 
males gain a mating equally fast or faster than young males in Drosophila pseudoobscura (Dhole \& Pfennig 2014) and D. bipectinata (Somashekar \& Krishna 2011).

While we commonly think of an individual ageing with all traits declining synchronously, recent studies found asynchrony in ageing rates in for example wild Soay sheep (Ovis aries, Hayward et al. 2015) and for female but not male fowl (Cornwallis et al. 2014). This might be due to these traits having different energy requirements or incur different trade-off costs resulting in different ageing rates. This highlights that in studies of ageing we need to investigate traits separately to gain an integrated view on how an organism ages and to understand the factors that influence senescence rates across traits and subsequently reproductive output.

\section{Senescence of male post-mating traits}

Apart from the ability to gain a mating, often the reproductive gain from these mating interactions is reduced, when older males exhibit a declining ability to father offspring. Reproduction is a multi-step process and several of these steps occur post mating, culminating in successful fertilisation and offspring production. Several of these steps are mediated by components of the male ejaculate (Box 1). Decreases in male ability to elicit favourable female post-mating responses result in reduced paternity shares for older males (McDonald et al. 2017, Ruhmann et al. 2018).

Post-mating effects of male ageing are understudied in the wild (but see Kim et al. 2011), particularly in small, short-lived species, and most of our insight comes from captivity. There is accumulating evidence that males suffer from senescence of post-mating reproductive traits in some species (e.g. the cricket Allonemobius socius, Marshall et al. 2009; the blue-footed booby, Kim et al. 2011; the houbara bustard, Preston et al. 2015) but this is not universal (for flies e.g. Santhosh \& Krishna 2013, Dhole \& Pfennig 2014, Herrera-Cruz et al. 2018).
One possibility could be that studies not always tested males at the far end of a species age range. We aimed to include studies that span a significant age range across a male's lifespan, but a large number of studies do not report lifespan concurrently for their species under study. When possible we tried to find other papers reporting lifespan (Supplementary Table 1, see section on supplementary data given at the end of this article), though we are aware that differences in study conditions can affect lifespan estimates. This is problematic, when no effect is found and we cannot be sure this is due to a lack of including truly old males, when estimating effect sizes and when we want to compare across species to be able to identify similar stages of a male's life.

The most common measure of male post-mating success is the number of eggs or offspring a male's mating partner produces. In most species including birds, insects and mammals, these numbers are reduced after mating with a senescing male (Marshall et al. 2009, Kim et al. 2011, Rezaei et al. 2015, Koppik \& Fricke 2017, Ruhmann et al. 2018; for a comprehensive review on vertebrates in the wild see Lemaitre \& Gaillard 2017), but exceptions exist (Santhosh \& Krishna 2013, Dhole \& Pfennig 2014). Only few studies provide a more detailed view by reporting egg numbers and egg-to-adult survival/ hatching success (Preston et al. 2015, Koppik \& Fricke 2017, Ruhmann et al. 2018) or sperm competition success (Koppik \& Fricke 2017, Ruhmann et al. 2018). These measurements though can give valuable insight into the mechanisms of male reproductive ageing.

In polyandrous species one male strategy to avoid post-mating competition between male ejaculates is to reduce the likelihood of female remating. Senescing D. melanogaster males can become increasingly poor in inducing this refractory period in females (Koppik \& Fricke 2017, Koppik et al. 2018, Ruhmann et al. 2018). Cumulative losses occur when males additionally have a diminished ability to compete over fertilisations as seen in the fowl or the vinegar fly (Dean et al. 2010,

\section{Box 1: Seminal fluid proteins}

Seminal fluid proteins (Sfps) occur in many internally and externally fertilising species, where they aid male reproductive success through their contributions to various processes, such as sperm capacitation, sperm competition and fertilisation (reviewed in Poiani 2006). Though the majority of Sfps is secreted by the various male accessory glands, other reproductive tract tissues can contribute to the non-sperm fraction of the ejaculate (McGraw et al. 2015). Drosophila melanogaster is an important model in research on the identification and function of Sfps (Box 2), but the number of species in which we have investigated Sfps is steadily increasing (for a review see Avila et al. 2011), whereby in insect taxa, they play an important role in a multitude of physiological and behavioural female post-mating changes. In mosquitoes Sfps influence for example, female egg maturation and host-seeking behaviour (reviewed in Klowden 1999), processes which are also important for their role as vectors of various diseases, and 198 respectively 93 Sfps have been identified in Aedes albopictus (Boes et al. 2014) and A. aegypti (Sirot et al. 2011). Studies on Sfp identity and function also extend to various vertebrate species (e.g. Druart et al. 2013 for a comparison across seven domestic mammalian species). In humans, 923 seminal fluid proteins have been identified through mass spectrometry (Pilch \& Mann 2006). Common functions include sperm capacitation, contribution to sperm motility and survival within the female reproductive tract (Poiani 2006) and thus are important aids to male fertilisation success. Beyond fertilisation, seminal fluid can even impact offspring (metabolic) phenotype as recently shown in mice (Watkins et al. 2018). Investigations on Sfps in birds are relatively scarce, but a recent study in Red Junglefowl Gallus gallus reports a complex seminal fluid cocktail with 1141 Sfps (Borziak et al. 2016). Seminal fluid proteins are ubiquitous in sexually reproducing animals and, while their importance in reproduction already is apparent, many novel functions are likely to be discovered when we add more species to this list. 
Koppik \& Fricke 2017, McDonald et al. 2017, Koppik et al. 2018, Ruhmann et al. 2018). Since polyandry is common, considering senescing males' success in competitive situations is important to fully understand reproductive ageing in males, and thus calls for more studies integrating these aspects of male reproduction.

\section{Mechanisms of male reproductive ageing}

In males of advancing age, ejaculate size has been observed to decrease (e.g. the houbara bustard, Chantepie et al. 2015) as well as increase (e.g. the butterfly Bicyclus anynana, Kehl et al. 2015; the Glanville fritillary butterfly, Duplouy et al. 2018), with several conceivable explanations. Older males could actively alter their investment into each mating, possibly increasing it due to their lower residual reproductive value. Alternatively, the tissues producing the ejaculate ages, resulting in fewer products available and an overall smaller and less effective ejaculate which may have a variety of consequences for male reproductive success (see Fig. 1 for a graphical outline using D. melanogaster as an example). As the ejaculate is made up of sperm and the non-sperm components, we outline how somatic and germline senescence affect fertilisation success by considering age-dependent changes in sperm quality and the non-sperm fraction of an ejaculate separately for a comprehensive understanding of the consequences for male reproductive success. The two ejaculate fractions might show independent responses to male ageing as they are produced by different tissues. The testis and its germline are well protected with an expensive DNA repair and maintenance machinery (Maklakov \& Immler 2016), which should at least slow germline ageing, while we would potentially expect less protection in the somatic reproductive tissues.

\section{Sperm and testes ageing}

To date theoretical considerations and empirical investigations into reproductive ageing of post-mating traits are mostly limited to sperm senescence (reviewed in Reinhardt 2007), distinguishing between two phases: pre- versus post-meiotic senescence (reviewed in Pizzari et al. 2008). Sperm ageing can be influenced by male actuarial ageing (pre-meiotic) but also be independent, when mature (post-meiotic) sperm age while being stored for extended periods, either within the male before ejaculation or within the female storage organs. Pre-meiotic ageing occurs due to the progressive deterioration of the spermatogenic machinery resulting in sperm not able to perform their primary goal of delivering their genetic contribution to the ova. Secondly, pre-meiotic sperm senescence is driven by the accumulation of mutations and might depend on the number of germline divisions. Deterioration of sperm genetic quality can have transgenerational effects and lead to unhealthy offspring and/or offspring carrying compromised germlines. Despite Pizzari and colleagues having reviewed the basis for sperm ageing more than 10 years ago, the field has only slowly gained insight into the functional and genetic basis of sperm ageing and/or factors that increase or slow its progression.

Longitudinal studies following individual males allow repeated sampling of ejaculate characteristics and sperm traits across their lifespan. This approach provides great insight into dynamic changes with age at the individual level, but is less amenable to wild animals and so far has been done for captive animals only. In houbara bustards, this approach revealed severe deteriorations of male ejaculate quality with advancing age, for example, ejaculate size decreased, as did sperm viability (Chantepie et al. 2015). Additionally, ejaculated sperm numbers dropped by over $60 \%$ and the probability of producing sperm with severely impaired motility more than doubled (Preston et al. 2011). Similarly, following a group of captive zebrafish males (Danio rerio) over 31 months revealed fewer and fewer males to produce sperm. A decrease in sperm numbers was already observed early on, while a decline in motility occurred later in life (Johnson et al. 2018). With velocity being a key predictor of fertilisation success in external fertilisers, this should negatively impact male reproduction. In both cases, ejaculates could be sampled directly and repeatedly from individual males and revealed fewer and slower sperm to be produced with advancing male age. However, in both studies, males were abstinent between collections, thus ejaculate age was dependent on sampling interval. Ejaculate age affects sperm swimming speed in the guppy Poecilia reticulate, with sperm from a young ejaculate swimming faster (Gasparini et al. 2014), while the opposite effect was observed in the eastern mosquito fish Gambusi holbrooki (Vega-Trejo et al. 2019) and hence results from longitudinal studies might depend on the length of sampling intervals. Disentangling male age effects on ejaculate quality from ejaculate age thus would be informative, but is not trivial, particularly in animals where an ejaculate represents a fraction of the sperm and non-sperm components in storage. To clearly separate male age from ejaculate age effects, a male needs to be stripped first of his entire ejaculate to ensure a uniformly aged ejaculate and not a mix of freshly produced versus old products still in storage. Such an approach is promising in animals, where ongoing spermatogenesis is the norm and thus older males are as capable as young ones to deliver a 'fresh' ejaculate.

The observation for ejaculates of ageing males to contain fewer sperm is not uncommon (Supplementary Table 1) and indicates that also sperm production might be slowed (Vega-Trejo et al. 2019). Indeed in $D$. melanogaster, fewer germ line stem cells can be found in the testis stem cell niche with advancing male age (Boyle et al. 2007). The stem cells maintain their 


\section{A Male reproductive tract}

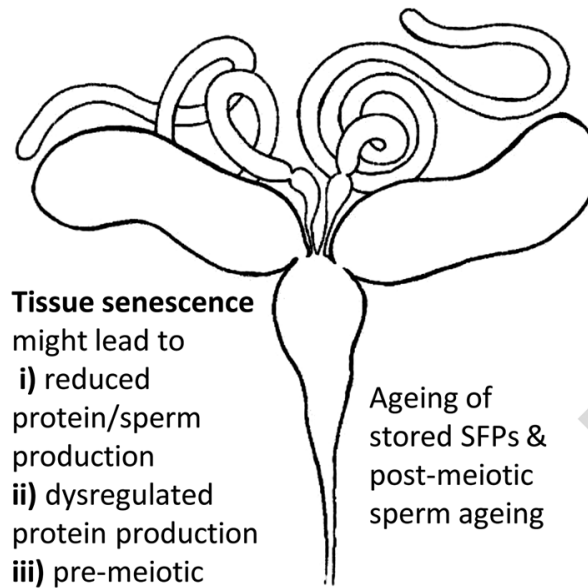

Reduced mating rate

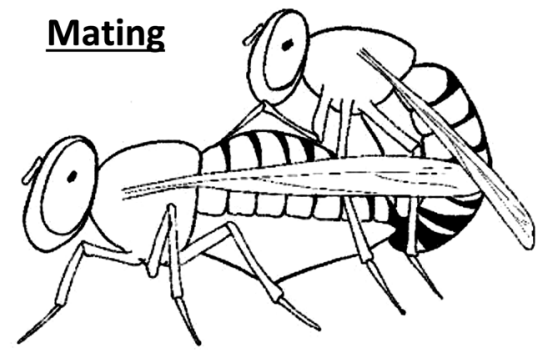

iii) pre-meiotic

sperm ageing

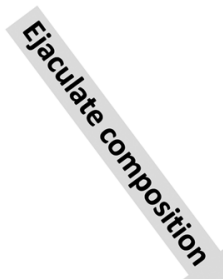

Male senescence

might change

transfer of

sperm/SFPs

Female choice might cause shorter transfer times and/or increased expel of sperm/SFPs

\section{Ejaculate quality \& quantity}

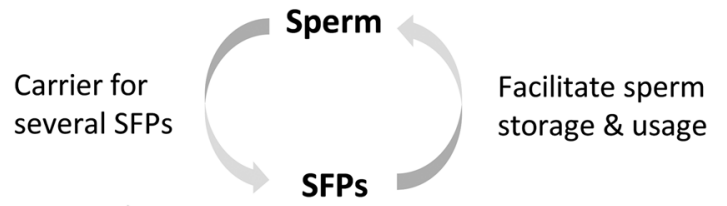

immune system

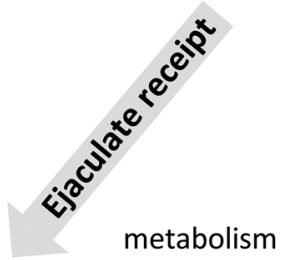

uterine conformational

changes

oviposition

\section{Female post-mating} response

feeding and

sleeping patterns

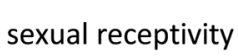

ovulation

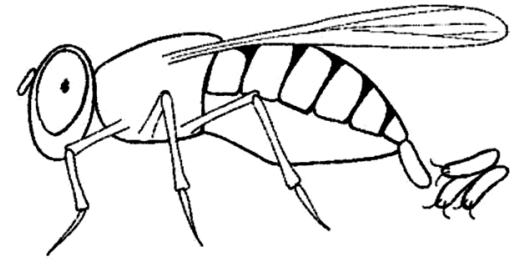
sperm storage

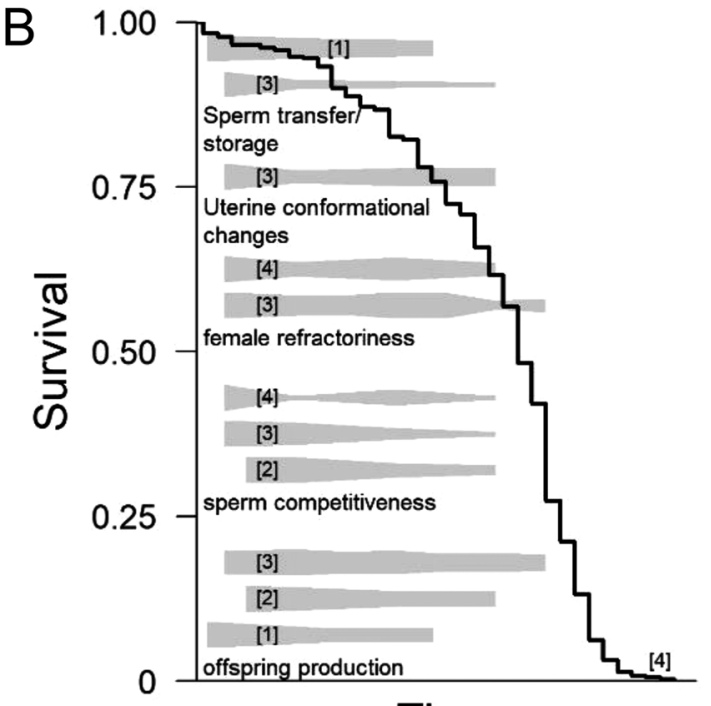

Time

Figure 1 (A) Schematic overview over possible routes via which Sfps and sperm in senescing Drosophila melanogaster males might influence female post-mating responses and thus male reproductive success. Information on the identity and function of various Sfps can be found in Box 2. (B) Graph shows survival (solid line) and expression of Sfp-mediated post-mating traits (in grey with changes in the magnitude of expression over time depicted by the thickness of the bar) in ageing $D$. melanogaster males over time. Mean survival time for males is assumed to be 65 days for data set [1] and 41.2 days for data sets [2], [3] and [4]. Data are derived from: [1] Rezaei et al. (2015), [2] Koppik and Fricke (2017), [3] Ruhmann et al. (2018), [4] Koppik et al. (2018). 
ability to divide, but do so slower leading to a decreased spermatogenesis and thinner testes (Boyle et al. 2007). A reduced sperm production can translate into fewer sperm delivered to the female, matching observations of $D$. melanogaster females having fewer sperm in storage after mating to older males (Rezaei et al. 2015, Ruhmann et al. 2018). Transfer of less sperm though is not the rule, as the prospect of few remaining mating opportunities can enhance male investment in reproduction. Accordingly, older males transfer more sperm at mating than younger ones in for example $D$. bipectinata (Santhosh \& Krishna 2013) or the butterfly B. anynana, in singly but not double mated males (Kehl et al. 2015). Also in humans, sperm concentration does not decline with age, but overall semen volume decreases (Johnson et al. 2015). However, increased or similar investments can be counteracted by other age-related changes as for example $B$. anynana females mated to younger males display higher fecundity than those mated to older ones despite older males' increased investment (Kehl et al. 2015), indicating that sperm numbers transferred is not a guarantee for reproductive success. Similarly in Homo sapiens sperm from older males were less motile and showed abnormal morphology (reviewed in Johnson et al. 2015).

Apart from the ability to produce and transfer sperm, viability is another commonly used measure of sperm ageing. So far, no consistent age-dependent pattern emerges for sperm viability (Supplementary Table 1). In general, sperm viability is higher in polyandrous species (Hunter \& Birkhead 2002) due to selection pressures to maintain high fertilisation capacity under sperm competition. Hence, it is worthwhile to consider mating system in these assays as monandrous males might suffer stronger from an age-dependent decline in sperm viability than polyandrous ones and this might partly explain the variation in sperm viability data.

Increased accumulation of DNA damage with male age was reported in for example humans (Wyrobek et al. 2006, Kong et al. 2012) and old blue-footed booby males (Velando et al. 2011). The rate of mutation accumulation is predicted to be influenced by the number of germline stem cell divisions (Bartosch-Härlid et al. 2003). On a mechanistic level, sperm damage due to reactive oxygen species (ROS) has found much traction (reviewed in Reinhardt 2007). ROS production is tightly linked to metabolic activity, as it is a byproduct of mitochondrial energy production (Reinhardt 2007). In B. anynana both oxidative damage and antioxidative defence improved with male age (Kehl et al. 2015). In contrast, in the fowl, levels of seminal antioxidants were lower by $37 \%$ in old compared to young males potentially explaining the higher DNA damage found in older males (Noguera et al. 2012). Particularly old polyandrous males, who underwent multiple rounds of divisions, should possess more DNA damage, unless they also invest more in DNA repair and maintenance mechanisms. This damage has evolutionary consequences due to its transgenerational effects and potential to persist in the germline, but to our knowledge a systematic investigation into age, male mating system/activity and DNA damage is lacking.

Sperm ageing has received much attention and while data are accumulating, we still have large gaps in our understanding. First, the distinction between pre-and post-meiotic sperm ageing is not trivial when length of post-meiotic storage inside the male is not controlled for. Also often single measures of age-dependent changes in sperm number or morphology are not linked to fertilisation ability (for e.g. by Moller et al. 2009, Gasparini et al. 2014, Herrera-Cruz et al. 2018). It is often implicitly assumed that age-dependent changes would result in a diminished fertilisation success; however, this is not necessarily the case as in some species like D. bipectinata and D. pseudoobscura, older males have higher reproductive success than younger males. Furthermore, the loss of male fecundity in D. melanogaster cannot always be explained by a decline in sperm numbers, as similarly a lack of seminal fluid proteins can lead to low fertility despite the presence of fertile sperm (Chow et al. 2015). Hence, in the next section, we will review the consequences of senescence of the non-sperm fraction of an ejaculate.

\section{Ageing of the non-sperm fraction of the ejaculate}

Across the animal kingdom, the non-sperm part of the seminal fluid contributes to reproductive success and shapes male-female interactions up until fertilisation. Some authors even suggest their absence might contribute to low implantation success in assisted reproductive technology (McGraw et al. 2015). The nature and function of seminal fluid proteins (Sfps) are well studied in D. melanogaster (Box 2), but are also known for other animals (Box 1). Ageing of the tissues that produces the non-sperm fraction and related quality changes has received far less attention in the study of male reproductive ageing. We here highlight that the onset and course of male reproductive senescence is significantly shaped by age-dependent changes in the production, transfer and action of Sfps (see also Bastos et al. 2017, Fig. 1).

While testis size often seems to decrease with male age (Supplementary Table 1), the opposite pattern is often found for the accessory gland (AG), the main Sfp producing tissue in insects (e.g. in flies, Rezaei et al. 2015, Reyes-Hernandez \& Perez-Staples 2017; but see Mazeed and Mohanny, 2010 for honeybees). Whether these size changes improve male fecundity is unclear, as increased AGs contain more protein in some (e.g. $D$. bipectinata and D. melanogaster, Santhosh \& Krishna 2013, Rezaei et al. 2015), but not all species (e.g. the Mexican fruit fly, Herrera-Cruz et al. 2018) and do not indicate how much protein is available at mating (Rezaei et al. 2015). The length of copulation duration 


\section{Box 2: Seminal fluid proteins in Drosophila melanogaster}

In the fruit fly D. melanogaster, the structure and function of seminal fluid proteins (Sfps) have been intensively studied (reviewed in Chapman \& Davies 2004). A large fraction of these Sfps are produced and stored in the male accessory gland, and thus are referred to as accessory gland proteins (Acps, reviewed in Ravi Ram \& Wolfner 2007). To date, about 200 different Sfps have been described in D. melanogaster and at least 138 of those are transferred to the female during mating (Findlay et al. 2008). Many Sfps have a profound effect on female behaviour and physiology (referred to as female post-mating response) elevating a male's reproductive success (Chapman \& Davies 2004). The most prominently studied Sfp is sex peptide (SP), a short (36 amino acids long) peptide (Kubli 2003), for which the corresponding receptor in the female has been identified (SPR, Yapici et al. 2008). Upon ejaculate transfer, SP binds to sperm and is later gradually released in mated females, thus sustaining its effect long term ( 1 week) compared to other Sfps (Peng et al. 2005). At the same time, other Sfps, for example Acp36DE, facilitate female sperm storage (Avila \& Wolfner 2009), which highlights the intricate relationship between Sfps and sperm. Most Sfps act short term $(\sim 24 \mathrm{~h})$ and ready the female reproductive system. Both Acp36DE and ovulin (Acp26Aa) facilitate the passage of the eggs. Acp36DE does so by mediating uterine conformational changes (Avila \& Wolfner 2009), while ovulin simultaneously relaxes the oviduct musculature (Rubinstein \& Wolfner 2013) causing a short-term boost in ovulation (Heifetz et al. 2005). SP influences female physiology in many ways, for example, by altering feeding intensity (Carvalho et al. 2006) and sleeping patterns (Isaac et al. 2010). Importantly, it enhances oogenesis leading to a substantial increase in egg laying after mating (Soller et al. 1997).

Apart from physiological changes also behaviour is affected and one important function of SP is the inhibition of female sexual receptivity (Chapman et al. 2003), which aids male reproductive success by avoiding sperm competition within the female reproductive tract. Short-term inhibition of female remating is further aided by PEBII, an Sfp associated with the mating plug produced by D. melanogaster males (Bretman et al. 2010). However, sperm competition within the female reproductive tract frequently occurs in $D$. melanogaster and the non-sperm fraction of the ejaculate is crucial to ensure that a male's sperm is represented in the fertilisation set to gain a significant paternity share (Fiumera et al. 2005, 2007). Due to their diverse roles in determining male reproductive success, we hypothesised several pathways through which Sfps might contribute to male reproductive ageing in D. melanogaster (summarised in Fig. 1).

is often used as an indirect indication of investment into an ejaculate. In D. pseudoobscura copulation duration roughly doubled from 2-4 to 14-19-day-old males and these older males indeed induced a higher egg-laying rate (Dhole \& Pfennig 2014), indicating that more of the relevant Sfps were transferred as observed in D. bipectinata (Santhosh \& Krishna 2013). Ageing D. melanogaster males, on the other hand, transfer smaller ejaculates despite longer mating durations and larger AGs (Koppik et al. 2018). Hence, while copulation duration can be a good indicator of male investment, some caution is warranted. Additionally, senescing AGs in D. melanogaster lose their integrity and eventually individual cells at the tip of the gland exit the cell layer and entire delaminated cells are transferred to the female (Leiblich et al. 2012), bearing testimony to the profound structural changes of the tissue itself.

Senescence of the AG manifests at the phenotypic level as males failing to induce the appropriate female post-mating responses to Sfps. In the cricket Allonemobius socius, a reduced transfer of the Sfp ejaculate serine protease with advancing male age (8-40-day-old males) can be linked to a reduced eggoutput in females (Marshall et al. 2009). Similarly, ageing D. melanogaster males (up to 6 weeks of age) decline in their ability to induce Sfp-mediated phenotypic post-mating changes, which together explain a large fraction of the observed lower reproductive success of older males, that was particularly evident when males encountered competition (Ruhmann et al. 2018). While only based on two Sfps, quantification of Sfp transfer revealed a mixed picture of age-dependent changes and no clear linear decline (Ruhmann et al. 2018), despite reduced expression for individual Sfp genes with age (Koppik \& Fricke 2017). These are first steps and should be complemented by larger scale transcriptome and/ or proteome studies. Assays of age-dependent changes in post-translation processes might prove equally informative, as this might explain the initial discrepancy between these first studies on Sfp expression, transfer and male phenotypic ability to elicit beneficial responses.

Different Sfps are often produced by distinct tissues or specialised cells within a tissue, with differential agedependent declines in functionality potentially resulting in compositional changes. Particularly, senescence in regulatory mechanisms might lead to a dysregulated production of Sfps resulting in a sub-optimal seminal fluid composition. There is surprisingly little information on age-dependent compositional changes. One notable exception is the comparison of fowl ejaculates, and while compositional changes were found, again there were no simple age-dependent dynamics, as there was a strong distinction due to male age and sperm velocity (Borziak et al. 2016). Particularly, old males that retained high sperm velocity had a distinct Sfp signature, while those old males with slow sperm clustered closely with younger males (Borziak et al. 2016). While this is the first direct test, other studies hint at the possibility of compositional changes, but either have only explored a limited set of Sfps (Koppik \& Fricke 2017, Ruhmann et al. 2018) or covered a limited age range (Simmons et al. 2014). Extending and broadening these studies using proteomics, allowing for direct quantification of proteins present and linking those insights with phenotypic data will be a fruitful avenue to our understanding of potential limitations ageing males encounter in producing a functional ejaculate. Here, longitudinal studies might prove particularly useful, by directly describing the trajectory of Sfp changes for individual males across their lifespan. This approach though can currently still 
be technically and/or financially challenging. Species producing spermatophores might be a worthwhile study system as spermatophores are easily accessible and can be repeatedly sampled from the same individual.

\section{Seminal fluid and sperm interactions}

We so far considered the consequences of senescence of sperm and the non-sperm part of an ejaculate and the tissues that produce them separately. However, these two fractions interact, as the seminal fluid can have a protective function maintaining sperm viability (Simmons \& Beveridge 2011) or aid sperm maturation (e.g. see Borziak et al. 2016 and literature within) and is also discussed as one possible explanatory factor in age-related declines in human fertility (Sartorius and Nieschlag 2010). Here we summarise the limited knowledge of the joint effects of age-related changes for both ejaculate fractions and highlight that this is a promising avenue for future research.

As outlined in Box 2, Sfps aid sperm in reaching the sperm storage organs in D. melanogaster with older males less able to elicit the uterine transformational changes mediated by Acp36DE and as a consequence fewer sperm were found in storage shortly after mating (Ruhmann et al. 2018). Additionally, several of the Sfps are known to bind to the sperm tail (Peng et al. 2005), fewer sperm being transferred and/or stored thus also means fewer of those proteins being available to the female. The impact of senescent changes in these Sfpsperm interactions on male reproductive success clearly need further investigations.

The only other system, in which some of these dynamics between senescence in sperm traits and age-dependent changes in seminal fluid proteins have been investigated, is the fowl. Here, older males that retain high sperm velocity, a trait known to positively affect fertilisation success in this species, possessed a distinct Sf proteome (Borziak et al. 2016), indicating that males might be able to adjust their Sfp composition to maintain high sperm velocity and hence fertilisation rate. Though, clearly not all males were able to do so. In an independent study, older males displayed a low level of protective seminal antioxidants that was associated with a tendency to reduce sperm motility and sperm carried a signature of oxidative DNA damage (Noguera et al. 2012), indicating that the protection is leaky. As old males retained a high level of plasma antioxidants that was positively correlated with male comb size, a trait important for mating success, the authors speculated whether males trade-off post-mating success for premating success as they age (Noguera et al. 2012). This opens up the possibility for condition-dependent ageing processes in this species which might explain why not all males in the previous study displayed a Sf proteome that facilitated high sperm velocity at old age. Given the high number of Sfps that are involved in sperm capacitation, motility and viability at least in mammals (reviewed in Poiani 2006), Sfps are likely to play a key role in shaping these sperm traits in ageing males and thus deserve further investigation.

\section{Interactions between the female reproductive tract and male ageing sperm}

While male reproductive ageing and sperm ageing can compromise sperm genetic quality, females might take an active role and ameliorate damage. Support for this notion comes from the observation that Drosophila females can repair DNA damage in sperm (Vogel et al. 1985, Agrawal \& Wang 2008). Apart from DNA repair, females can prevent negative effects of post-meiotic sperm ageing by eliciting extra copulations as seen in the black-legged kittiwake (Rissa tridactyla) (White et al. 2008) or reject copulations with old males, particularly if males reliably advertise the extent of their sperm damage as in the blue-footed booby (Velando et al. 2011). Additionally, female physiological adaptations aid sperm viability as observed in birds and the cricket Gryllus bimaculatus (Breque et al. 2003, Ribou \& Reinhardt 2012) or sperm movement patterns in barn swallows Hirundo rustica (Moller et al. 2009) and alleviate the effects of post-meiotic sperm ageing. This indicates that females might actively alter sperm metabolism across a wide range of taxa and potentially protect sperm from degradation or exert cryptic female choice, whereby in zebrafish sperm selection can benefit offspring fitness (Alavioon et al. 2017).

\section{Factors affecting reproductive ageing}

A host of factors are known to alter the speed of actuarial ageing; however, we have limited insight, whether these factors have a similar effect on reproductive ageing. Rates of senescence are modulated by the lifespan - fecundity trade-off, whereby increased investment in early reproduction often accelerates somatic ageing (Kim et al. 2011, Cornwallis et al. 2014, Lemaitre et al. 2015), but also reproductive ageing (Preston et al. 2011). The longstanding underlying premise being that reproduction and somatic maintenance compete over resource allocation. More recently, hyperfunction (Blagosklonny 2012) - the idea that cellular processes optimal during development and early life continue to run at high levels during late life, where they cause harm and thus promote ageing - presents an alternative explanation for senescence (Gems \& Partridge 2013). A recent study in C. elegans supports this idea: reduction of insulin/IGF-1 nutrient sensing signalling in adult nematodes simultaneously improved late-life reproduction (of mated worms) and longevity without any decline in total reproduction (Lind et al. 2019). Moreover, offspring of those treated worms had increased reproduction compared to worms from parents with a normal level of insulin/IGF-1 signalling, 
thus showing that longevity and Darwinian fitness can be improved at the same time (Lind et al. 2019). Studying the genetic basis of reproductive ageing in $C$. elegans Wang et al. (2014) found 32 genes to extend reproductive lifespan, many of which interacted with either the insulin/IGF 1 or the TGF $ß$ pathways. In this hermaphroditic animal equally many affected oocyte senescence (19 genes) as well as senescence of male function (11 genes), but curiously this did not affect spermatogenesis (Wang et al. 2014). These pathways also have a prominent role in affecting actuarial ageing and this might be part of a general and conserved link underlying the apparent fecundity-longevity tradeoff. Illuminating the genetic factors of reproductive ageing will be fruitful future endeavours to pinpoint the pathways underpinning reproductive ageing.

Similar to actuarial ageing, reproductive ageing could potentially also decelerate under caloric restriction, when simultaneously the reproductive tissue is protected from physiological decline. Indeed, dietary restriction attenuated the age-related decline in male reproductive senescence in $D$. melanogaster by enhancing male germline stem cell maintenance and halting the age-related depletion (Mair et al. 2010). While at the phenotypic level, diet had little influence on age-dependent changes in insemination success in the Mexican fruit fly A. ludens (Harwood et al. 2015). The question whether males can delay reproduction and reallocate resources towards increased survival in times of scarcity and simultaneously decelerate reproductive ageing deserves further investigations.

Another fruitful avenue of potential modulators of reproductive ageing is the social environment whereby intra-sexual competition (RodriguezMunoz et al. 2019) or the mere perception of mates in the environment (Gendron et al. 2014) can cause increased mortality and senescence in pre-mating traits (Rodriguez-Munoz et al. 2019). This is in line with observations that the socio-sexual environment leads males to allocate more resources into reproduction when rivals are present (Bretman et al. 2011), with males held in isolation showing a slower reduction in lifespan (Leech et al. 2017). In D. melanogaster, male mating history did not accelerate reproductive ageing for pre-mating traits, but caused a distinct pattern for post-mating traits (Koppik et al. 2018). For these latter traits, prior mating opportunities proved beneficial in young males but neutral or negative in older males, potentially due to different reproductive investments and a trade-off between early and late reproductive efforts.

\section{Open questions and outlook}

One point became obvious from our review of male reproductive ageing across animal taxa: comparisons among different studies are often hampered because authors fail to report lifespans in their experimental population. This oversight makes it difficult to appropriately classify investigated age ranges. Hence, future studies should routinely report the average and maximal lifespan of their species. Ideally, measures of lifespan should be conducted simultaneously in the experimental cohort, as lifespan estimates can vary critically with experimental conditions. Additionally, cohort-based experiments might not be ideal when investigating senescence patterns. Since the selective appearance and disappearance of individuals with different reproductive strategies (Kim et al. 2011) and/ or genetic quality (Torres et al. 2011) can mask or inflate ageing patterns when considering the entire cohort (Bouwhuis et al. 2009, Dugdale et al. 2011), calling for more studies following individuals across their lifespan. Longitudinal studies give greater insight into possible trade-offs, the shape and rate of age-dependent changes and allow better control for selective disappearance and the possible reasons behind it.

Here, we have outlined changes occurring in sperm and seminal fluid with male age and summarised reproductive trait expression in senescing males. While data are accumulating on each of the aforementioned aspects, studies integrating several or all facets of male reproduction in ageing individuals are still scarce (but see Marshall et al. 2009). Often, it is technically challenging to access all male components that contribute to reproductive success and the techniques required to thoroughly examine them might be very different for sperm and Sfps. However, given the intricate relationship between sperm and the nonsperm fraction of the ejaculate, we suggest that more integrated studies are necessary to fully understand male reproductive senescence. Reports on changes in sperm with male age outnumber those investigating a male's Sfp complement by far, but, with transcriptomics and proteomics becoming more accessible, this might soon start to change.

Another promising and exciting avenue is revealing how ejaculates of ageing males affect the multitude of interactions with female physiology. We already highlighted that females might actively alter ejaculate characteristics and through (cryptic) choice and investment decisions might mitigate or exaggerate effects of male senescence. Disentangling male age from female choice and elucidating the underlying mechanisms will be exciting future research programmes. Hence, many open questions remain into the ecological and evolutionary causes and consequences of male reproductive ageing.

\section{Supplementary data}

This is linked to the online version of the paper at https://doi.org/10.1530/REP-18-0579. 


\section{Declaration of interest}

The authors declare that there is no conflict of interest that could be perceived as prejudicing the impartiality of this review.

\section{Funding}

C F was supported by the DFG through their Heisenberg program (FR 2973/8-1) and a grant within the research unit FOR 2281 (FR 2973/7-1).

\section{Acknowledgements}

The authors want to thank the review editors for the opportunity to write this review and anonymous reviewers for helpful suggestions.

\section{References}

Agrawal AF \& Wang AD 2008 Increased transmission of mutations by lowcondition females: evidence for condition-dependent DNA repair. PLoS Biology 6 e30. (https://doi.org/10.1371/journal.pbio.0060030)

Alavioon G, Hotzy C, Nakhro K, Rudolf S, Scofield DG, Zajitschek S, Maklakov AA \& Immler S 2017 Haploid selection within a single ejaculate increases offspring fitness. PNAS 114 8053-8058. (https://doi. org/10.1073/pnas.1705601114)

Avila FW \& Wolfner MF 2009 Acp36DE is required for uterine conformational changes in mated Drosophila females. PNAS 106 15796-15800. (https://doi.org/10.1073/pnas.0904029106)

Avila FW, Sirot LK, LaFlamme BA, Rubinstein CD \& Wolfner MF 2011 Insect seminal fluid proteins: identification and function. Annual Review of Entomology 56 21-40. (https://doi.org/10.1146/annurevento-120709-144823)

Bartosch-Härlid A, Berlin S, Smith NG, Moller AP \& Ellegren H 2003 Life history and the male mutation bias. Evolution 57 2398-2406.

Bastos P, Freitas MJ, Gomes A, Vitorino R \& Fardilha M 2017 Unravelling the power of omics for the infertile aging male. Current Pharmaceutical Design 23 4451-4469. (https://doi.org/10.2174/1381612822666161018155247)

Blagosklonny MV 2012 Answering the ultimate question 'What is the Proximal Cause of Aging?' Aging 4 861-877. (https://doi.org/10.18632/ aging.100525)

Boes KE, Ribeiro JMC, Wong A, Harrington LC, Wolfner MF \& Sirot LK 2014 Identification and characterization of seminal fluid protein in the Asian Tiger Mosquito, Aedes albopictus. PLoS Neglected Tropical Diseases 8 e2946. (https://doi.org/10.1371/journal.pntd.0002946)

Borziak K, Alvarez-Fernandez A, Karr TL, Pizzari T \& Dorus S 2016 The seminal fluid proteome of the polyandrous red junglefowl offers insights into the molecular basis of fertility, reproductive ageing and domestication. Scientific Reports 6 35864. (https://doi.org/10.1038/ srep35864)

Bouwhuis S, Sheldon BC, Verhulst S \& Charmantier A 2009 Great tits growing old: selective disappearance and the partitioning of senescence to stages within the breeding cycle. Proceedings: Biological Sciences 276 2769-2777. (https://doi.org/10.1098/rspb.2009.0457)

Boyle M, Wong C, Rocha M \& Jones DL 2007 Decline in self-renewal factors contributes to aging of the stem cell niche in the Drosophila testis. Cell Stem Cell 1 470-478. (https://doi.org/10.1016/j.stem.2007.08.002)

Breque C, Surai P \& Brillard JP 2003 Roles of antioxidants on prolonged storage of avian spermatozoa in vivo and in vitro. Molecular Reproduction and Development 66 314-323. (https://doi.org/10.1002/mrd.10347)

Bretman A, Lawniczak MKN, Boone J \& Chapman T 2010 A mating plug protein reduces early female remating in Drosophila melanogaster. Journal of Insect Physiology 56 107-113. (https://doi.org/10.1016/j. jinsphys.2009.09.010)

Bretman A, Gage MJG \& Chapman T 2011 Quick-change artists: male plastic behavioural responses to rivals. Trends in Ecology and Evolution 26 467-473. (https://doi.org/10.1016/j.tree.2011.05.002)
Carvalho GB, Kapahi P, Anderson DJ \& Benzer S 2006 Allocrine modulation of feeding behavior by the sex peptide of Drosophila. Current Biology 16 692-696. (https://doi.org/10.1016/j.cub.2006.02.064)

Chantepie S, Robert A, Sorci G, Hingrat Y, Charmantier A, Leveque G, Lacroix F \& Teplitsky C 2015 Quantitative genetics of the aging of reproductive traits in the houbara bustard. PLOS ONE 10 e0133140. (https://doi.org/10.1371/journal.pone.0133140)

Chapman T \& Davies SJ 2004 Functions and analysis of the seminal fluid proteins of male Drosophila melanogaster fruit flies. Peptides $251477-$ 1490. (https://doi.org/10.1016/j.peptides.2003.10.023)

Chapman T, Bangham J, Vinti G, Seifried B, Lung O, Wolfner MF, Smith HK \& Partridge L 2003 The sex peptide of Drosophila melanogaster: female post-mating responses analyzed by using RNA interference. PNAS 100 9923-9928. (https://doi.org/10.1073/pnas.1631635100)

Chow CY, Avila FW, Clark AG \& Wolfner MF 2015 Induction of excessive endoplasmic reticulum stress in the Drosophila male accessory gland results in infertility. PLOS ONE 10 e0119386. (https://doi.org/10.1371/ journal.pone.0119386)

Cornwallis CK, Dean R \& Pizzari T 2014 Sex-specific patterns of aging in sexual ornaments and gametes. American Naturalist 184 E66-E78. (https://doi.org/10.1086/677385)

Dean R, Cornwallis CK, Lovlie H, Worley K, Richardson DS \& Pizzari T 2010 Male reproductive senescence causes potential for sexual conflict over mating. Current Biology 20 1192-1196. (https://doi.org/10.1016/j. cub.2010.04.059)

Dhole S \& Pfennig KS 2014 Age-dependent male mating investment in Drosophila pseudoobscura. PLOS ONE 9 e88700. (https://doi. org/10.1371/journal.pone.0088700)

Druart X, Rickard JP, Mactier S, Kohnke PL, Kershaw-Young CM, Bathgate R, Gibb Z, Crossett B, Tsikis G, Labas V et al. 2013 Proteomic characterization and cross species comparison of mammalian seminal plasma. Journal of Proteomics 91 13-22. (https://doi.org/10.1016/j. jprot.2013.05.029)

Dugdale HL, Pope LC, Newman C, MacDonald DW \& Burke T 2011 Agespecific breeding, success in a wild mammalian population: selection, constraint, restraint and senescence. Molecular Ecology 20 3261-3274. (https://doi.org/10.1111/j.1365-294X.2011.05167.x)

Duplouy A, Woestmann L, Zamorano J \& Saastamoinen M 2018 Impact of male condition on his spermatophore and consequences for female reproductive performance in the Glanville fritillary butterfly. Insect Science 25 284-296. (https://doi.org/10.1111/1744-7917.12424)

Findlay GD, Yi XH, MacCoss MJ \& Swanson WJ 2008 Proteomics reveals novel Drosophila seminal fluid proteins transferred at mating. PLoS Biology 6 e178. (https://doi.org/10.1371/journal.pbio.0060178)

Fiumera AC, Dumont BL \& Clark AG 2005 Sperm competitive ability in Drosophila melanogaster associated with variation in male reproductive proteins. Genetics 169 243-257. (https://doi.org/10.1534/ genetics.104.032870)

Fiumera AC, Dumont BL \& Clark AG 2007 Associations between sperm competition and natural variation in male reproductive genes on the third chromosome of Drosophila melanogaster. Genetics 176 12451260. (https://doi.org/10.1534/genetics.106.064915)

Gaillard JM \& Lemaitre JF 2017 The Williams' legacy: a critical reappraisal of his nine predictions about the evolution of senescence. Evolution 71 2768-2785. (https://doi.org/10.1111/evo.13379)

Gasparini C, Kelley JL \& Evans JP 2014 Male sperm storage compromises sperm motility in guppies. Biology Letters 10 20140681. (https://doi. org/10.1098/rsbl.2014.0681)

Gems D \& Partridge L 2013 Genetics of longevity in model organisms: debates and paradigm shifts. Annual Review of Physiology 75 621-644. (https://doi.org/10.1146/annurev-physiol-030212-183712)

Gendron CM, Kuo TH, Harvanek ZM, Chung BY, Yew JY, Dierick HA \& Pletcher SD 2014 Drosophila life span and physiology are modulated by sexual perception and reward. Science 343 544-548. (https://doi. org/10.1126/science.1243339)

Harwood JF, Chen K, Liedo P, Müller HG, Wang JL, Morice AE \& Carey JR 2015 Female access and diet affect insemination success, senescence, and the cost of reproduction in male Mexican fruit flies Anastrepha ludens. Physiological Entomology 40 65-71. (https://doi.org/10.1111/ phen.12089)

Hayward AD, Moorad J, Regan CE, Berenos C, Pilkington JG, Pemberton JM \& Nussey DH 2015 Asynchrony of senescence among phenotypic traits 
in a wild mammal population. Experimental Gerontology 71 56-68. (https://doi.org/10.1016/j.exger.2015.08.003)

Heifetz Y, Vandenberg LN, Cohn HI \& Wolfner MF 2005 Two cleavage products of the Drosophila accessory gland protein ovulin can independently induce ovulation. PNAS 102 743-748. (https://doi. org/10.1073/pnas.0407692102)

Herrera-Cruz M, Abraham S, Nunez-Beverido N, Flores-Estevez N, ReyesHernandez M, Alvarado M \& Perez-Staples D 2018 Male age and strain affect ejaculate quality in the Mexican fruit fly. Insect Science $25 \mathbf{7 0 3}$ 711. (https://doi.org/10.1111/1744-7917.12446)

Hunter FM \& Birkhead TR 2002 Sperm viability and sperm competition in insects. Current Biology 12 121-123. (https://doi.org/10.1016/s09609822(01)00647-9)

Isaac RE, Li C, Leedale AE \& Shirras AD 2010 Drosophila male sex peptide inhibits siesta sleep and promotes locomotor activity in the post-mated female. Proceedings: Biological Sciences 277 65-70. (https://doi. org/10.1098/rspb.2009.1236)

Johnson SL, Dunleavy J, Gemmell NJ \& Nakagawa S 2015 Consistent age-dependent declines in human semen quality: a systematic review and meta-analysis. Ageing Research Reviews 19 22-33. (https://doi. org/10.1016/j.arr.2014.10.007)

Johnson SL, Zellhuber-McMillan S, Gillum J, Dunleavy J, Evans JP, Nakagawa S \& Gemmell NJ 2018 Evidence that fertility trades off with early offspring fitness as males age. Proceedings of the Royal Society of London Series B 28520172174.

Jones OR, Scheuerlein A, Salguero-Gomez R, Camarda CG, Schaible R, Casper BB, Dahlgren JP, Ehrlen J, Garcia MB, Menges ES et al. 2014 Diversity of ageing across the tree of life. Nature 505 169-173. (https:// doi.org/10.1038/nature12789)

Kehl T, Beaulieu M, Kehl A \& Fischer K 2015 Old male sex: large ejaculate, many sperm, but few offspring. Behavioral Ecology and Sociobiology 69 1543-1552. (https://doi.org/10.1007/s00265-015-1966-1)

Kim SY, Velando A, Torres R \& Drummond H 2011 Effects of recruiting age on senescence, lifespan and lifetime reproductive success in a long-lived seabird. Oecologia 166 615-626. (https://doi.org/10.1007/s00442-0111914-3)

Klowden MJ 1999 The check is in the male: male mosquitoes affect female physiology and behavior. Journal of the American Mosquito Control Association 15 213-220.

Kong A, Frigge ML, Masson G, Besenbacher S, Sulem P, Magnusson G, Gudjonsson SA, Sigurdsson A, Jonasdottir A, Jonasdottir A et al. 2012 Rate of de novo mutations and the importance of father's age to disease risk. Nature 488 471-475. (https://doi.org/10.1038/nature11396)

Koppik M \& Fricke C 2017 Gene expression changes in male accessory glands during ageing are accompanied by reproductive decline in Drosophila melanogaster. Molecular Ecology 26 6704-6716. (https:// doi.org/10.1111/mec.14384)

Koppik M, Ruhmann H \& Fricke C 2018 The effect of mating history on male reproductive ageing in Drosophila melanogaster. Journal of Insect Physiology $111 \quad 16-24 . \quad$ (https://doi.org/10.1016/j. jinsphys.2018.10.003)

Kubli E 2003 Sex-peptides: seminal peptides of the Drosophila male. Cellular and Molecular Life Sciences 60 1689-1704. (https://doi. org/10.1007/s00018-003-3052)

Leech T, Sait SM \& Bretman A 2017 Sex-specific effects of social isolation on ageing in Drosophila melanogaster. Journal of Insect Physiology 102 12-17. (https://doi.org/10.1016/j.jinsphys.2017.08.008)

Leiblich A, Marsden L, Grandy C, Corrigan L, Jenkins R, Hamdy F \& Wilson C 2012 Bone morphogenetic protein- and mating-dependent secretory cell growth and migration in the Drosophila accessory gland. PNAS 109 19292-19297. (https://doi.org/10.1073/pnas.1214517109)

Lemaitre JF \& Gaillard JM 2017 Reproductive senescence: new perspectives in the wild. Biological Reviews of the Cambridge Philosophical Society 92 2182-2199. (https://doi.org/10.1111/brv.12328)

Lemaitre JF, Berger V, Bonenfant C, Douhard M, Gamelon M, Plard F \& Gaillard JM 2015 Early-late life trade-offs and the evolution of ageing in the wild. Proceedings: Biological Sciences 282 20150209. (https://doi. org/10.1098/rspb.2015.0209)

Lind MI, Ravindran S, Sekajova Z, Carlsson H, Hinas A \& Maklakov AA 2019 Experimentally reduced insulin/IGF-1 signaling in adulthood extends lifespan of parents and improves Darwinian fitness of their offspring. Evolution Letters 3 207-216. (https://doi.org/10.1002/evl3.108)
Mair W, McLeod CJ, Wang L \& Jones DL 2010 Dietary restriction enhances germline stem cell maintenance. Aging Cell 9 916-918. (https://doi. org/10.1111/j.1474-9726.2010.00602.x)

Maklakov AA \& Immler S 2016 The expensive germline and the evolution of ageing. Current Biology 26 R577-R586. (https://doi.org/10.1016/j. cub.2016.04.012)

Marshall JL, Huestis DL, Hiromasa Y, Wheeler S, Oppert C, Marshall SA, Tomich JM \& Oppert B 2009 Identification, RNAi knockdown, and functional analysis of an ejaculate protein that mediates a postmating, prezygotic phenotype in a cricket. PLOS ONE 4 e7537. (https://doi. org/10.1371/journal.pone.0007537)

Mazeed AM \& Mohanny KM 2010 Some reproductive characteristics of honeybee drones in relation to their ages. Entomological Research 40 245-250. (https://doi.org/10.1111/j.1748-5967.2010.00297.x)

McDonald GC, Spurgin LG, Fairfield EA, Richardson DS \& Pizzari T 2017 Pre- and postcopulatory sexual selection favor aggressive, young males in polyandrous groups of red junglefowl. Evolution 71 1653-1669. (https://doi.org/10.1111/evo.13242)

McGraw LA, Suarez SS \& Wolfner MF 2015 On a matter of seminal importance. BioEssays 37 142-147. (https://doi.org/10.1002/ bies.201400117)

Moller AP, Mousseau TA, Rudolfsen G, Balbotin J, Marzal A, Hermosell I \& De Lope F 2009 Senescent sperm performance in old male birds. Journal of Evolutionary Biology 22 334-344. (https://doi.org/10.1111/ j.1420-9101.2008.01650.x)

Noguera JC, Dean R, Isaksson C, Velando A \& Pizzari T 2012 Age-specific oxidative status and the expression of pre- and postcopulatory sexually selected traits in male red junglefowl, Gallus gallus. Ecology and Evolution 2 2155-2167. (https://doi.org/10.1002/ece3.300)

Nussey DH, Froy H, Lemaitre JF, Gaillard JM \& Austad SN 2013 Senescence in natural populations of animals: widespread evidence and its implications for bio-gerontology. Ageing Research Reviews 12 214-225. (https://doi.org/10.1016/j.arr.2012.07.004)

Peng J, Chen S, Buesser S, Liu H, Honegger T \& Kubli E 2005 Gradual release of sprm bound sex-peptide controls female postmating behavior in Drosophila. Current Biology 15 207-213. (https://doi.org/10.1016/j. cub.2005.01.034)

Pilch B \& Mann M 2006 Large-scale and high-confidence proteomic analysis of human seminal plasma. Genome Biology 7 R40. (https://doi. org/10.1186/gb-2006-7-5-r40)

Pizzari T, Dean R, Pacey A, Moore H \& Bonsall MB 2008 The evolutionary ecology of pre- and post-meiotic sperm senescence. Trends in Ecology and Evolution 23 131-140. (https://doi.org/10.1016/j.tree.2007.12.003)

Poiani A 2006 Complexity of seminal fluid: a review. Behavioral Ecology and Sociobiology 60 289-310. (https://doi.org/10.1007/s00265-0060178-0)

Preston BT, Saint Jalme M, Hingrat Y, Lacroix F \& Sorci G 2015 The sperm of aging male bustards retards their offspring's development. Nature Communications 6 6146. (https://doi.org/10.1038/ncomms7146)

Preston BT, Saint Jalme MS, Hingrat Y, Lacroix F \& Sorci G 2011 Sexually extravagant males age more rapidly. Ecology Letters 14 1017-1024. (https://doi.org/10.1111/j.1461-0248.2011.01668.x)

Ravi Ram K \& Wolfner MF 2007 Seminal influences: Drosophila Acps and the molecular interplay between males and females during reproduction. Integrative and Comparative Biology 47 427-445. (https:// doi.org/10.1093/icb/icm046)

Reinhardt K 2007 Evolutionary consequences of sperm cell aging. Quarterly Review of Biology 82 375-393. (https://doi.org/10.1086/522811)

Reyes-Hernandez M \& Perez-Staples D 2017 Mating senescence and male reproductive organ size in the Mexican fruit fly. Physiological Entomology 42 26-35. (https://doi.org/10.1111/phen.12160)

Rezaei A, Krishna MS \& Santhosh HT 2015 Male age affects female mate preference, quantity of accessory gland proteins, and sperm traits and female fitness in D. melanogaster. Zoological Science 32 16-24. (https:// doi.org/10.2108/zs140121)

Ribou AC \& Reinhardt K 2012 Reduced metabolic rate and oxygen radicals production in stored insect sperm. Proceedings: Biological Sciences 279 2196-2203. (https://doi.org/10.1098/rspb.2011.2422)

Rodriguez-Munoz R, Boonekamp JJ, Liu XP, Skicko I, Fisher DN, Hopwood P \& Tregenza T 2019 Testing the effect of early-life reproductive effort on age-related decline in a wild insect. Evolution 73 317-328. (https://doi. org/10.1111/evo.13679) 
Rubinstein CD \& Wolfner MF 2013 Drosophila seminal protein ovulin mediates ovulation through female octopamine neuronal signaling. PNAS 110 17420-17425. (https://doi.org/10.1073/pnas.1220018110)

Ruhmann H, Koppik M, Wolfner MF \& Fricke C 2018 The impact of ageing on male reproductive success in Drosophila melanogaster. Experimental Gerontology 103 1-10. (https://doi.org/10.1016/j.exger.2017.12.013)

Santhosh HT \& Krishna MS 2013 Relationship between male age, accessory gland, sperm transferred, and fitness traits in Drosophila bipectinata. Journal of Insect Science $\mathbf{1 3}$ 159. (https://doi. org/10.1673/031.013.15901)

Sartorius GA \& Nieschlag E 2010 Paternal age and reproduction. Human Reproduction Update 16 65-79. (https://doi.org/10.1093/humupd/ dmp027)

Simmons LW \& Beveridge M 2011 Seminal fluids affects sperm viability in a cricket. PLOS ONE 6 e17975. (https://doi.org/10.1371/journal. pone.0017975)

Simmons LW, Beveridge M, Li L, Tan YF \& Millar AH 2014 Ontogenetic changes in seminal fluid gene expression and the protein composition of cricket seminal fluid. Evolution and Development 16 101-109. (https:// doi.org/10.1111/ede.12068)

Sirot LK, Hardstone MC, Helinski MEH, Ribeiro JMC, Kimura $M$ Deewatthanawong P, Wolfner MF \& Harrington LC 2011 Towards a semen proteome of the dengue vector mosquito: protein identification and potential functions. PLoS Neglected Tropical Diseases 5 e989. (https://doi.org/10.1371/journal.pntd.0000989)

Soller M, Bownes M \& Kubli E 1997 Mating and sex peptide stimulates the accumulation of yolk in oocytes of Drosophila melanogaster. European Journal of Biochemistry 243 732-738. (https://doi.org/10.1111/j.14321033.1997.00732.x)

Somashekar K \& Krishna MS 2011 Evidence of female preference for older males in Drosophila bipectinata. Zoological Studies 50 1-15.

Torres R, Drummond H \& Velando A 2011 Parental age and lifespan influence offspring recruitment: a long-term study in a seabird. PLoS ONE 6 e27245. (https://doi.org/10.1371/journal.pone.0027245)

Vega-Trejo R, Fox RJ, Iglesias-Carrasco M, Head ML \& Jennions MD 2019 The effects of male age, sperm age and mating history on ejaculate senescence. Functional Ecology 33 1267-1279. (https://doi. org/10.1111/1365-2435.13305)

Velando A, Noguera JC, Drummond H \& Torres R 2011 Senescent males carry premutagenic lesions in sperm. Journal of Evolutionary Biology $\mathbf{2 4}$ 693-697. (https://doi.org/10.1111/j.1420-9101.2010.02201.x)

Vogel EW, Dusenbery RL \& Smith PD 1985 The relationship bewteen reaction kinetics and mutagenic action of monofunctional alkylating agents in higher eukaryotic systems. Mutation Research 149 193-207. (https://doi.org/10.1016/0027-5107(85)90026-0)

Wang MC, Oakley HD, Carr CE, Sowa JN \& Ruvkun G 2014 Gene pathways that delay Caenorhabditis elegans reproductive senescence. PLoS Genetics 10 e1004752. (https://doi.org/10.1371/journal.pgen.1004752)

Watkins AJ, Dias I, Tsuro H, Allen D, Emes RD, Moreton J, Wilson R, Ingram RJM \& Sinclair KD 2018 Paternal diet programs offspring health through sperm- and seminal plasma-specific pathways in mice. PNAS 115 10064-10069. (https://doi.org/10.1073/pnas.1806333115)

White J, Wagner RH, Helfenstein F, Hatch SA, Mulard H, Naves LC \& Danchin E 2008 Multiple deleterious effects of experimentally aged sperm in a monogamous bird. PNAS 105 13947-13952. (https://doi. org/10.1073/pnas.0803067105)

Wyrobek AJ, Eskenazi B, Young S, Arnheim N, Tiemann-Boege I, Jabs EW, Glaser RL, Pearson FS \& Evenson D 2006 Advancing age has differential effects on DNA damage, chromatin integrity, gene mutations and aneuplodies in sperm. PNAS 103 9601-9606. (https://doi.org/10.1073/ pnas.0506468103)

Yapici N, Kim YJ, Ribeiro C \& Dickson BJ 2008 A receptor that mediates the post-mating switch in Drosophila reproductive behaviour. Nature 451 33-37. (https://doi.org/10.1038/nature06483)

Received 8 November 2018

First decision 11 December 2018

Revised manuscript received 23 July 2019

Accepted 29 July 2019 